\title{
On Capillary Slowdown of Viscous Fingering in Immiscible Displacement in Porous Media
}

\author{
Prabir Daripa • G. Paşa
}

Received: 31 January 2007 / Accepted: 10 January 2008 / Published online: 12 February 2008

(C) Springer Science+Business Media B.V. 2008

\begin{abstract}
Hydrodynamic instability in immiscible porous media flows in the presence of capillarity is investigated here. The analysis and arguments presented here show that the slowdown of instabilities due to capillarity is usually very rapid which makes the flow almost, but not entirely, stable. The profiles of the stable and unstable waves in the far-field are characterized using a novel but very simple approach.
\end{abstract}

Keywords Stability $\cdot$ Capillarity $\cdot$ Immiscible flow $\cdot$ Porous media

\section{Introduction}

The dynamics and stability of immiscible fluid-fluid displacements in porous media is a subject of considerable interest, both from a fundamental point of view as a dynamical nonequilibrium process and from a technological point of view in industrial and environmental problems, such as oil recovery, irrigation, and filtration. This is particularly true in the context of enhancement of oil recovery from underground reservoirs. Such an enhanced oil recovery typically involves displacement of in-situ oleic phase by continuous injection of another fluid phase which may be immiscible (like water injection) or miscible (like $\mathrm{CO}_{2}$ or solvent injection) to the former. Stability considerations partly affect the degree of effectiveness of these methods (see Craig 1971; Daripa et al. 1988; Stalkup 1989). There are aspects related to the properties of porous medium matrix (as opposed to physical phenomena such as viscous fingering) which can be controlled to enhance oil recovery in some types of reservoirs. For example, in Al-Hadhrami and Blunt (2001) wettability of the matrix is controlled thermally to improve oil recovery in fractured reservoirs. To this end, it is worth citing the book of

P. Daripa $(\bowtie)$

Department of Mathematics, Texas A\&M University, College Station, TX 77843, USA

e-mail: prabir.daripa@math.tamu.edu

G. Paşa

Institute of Mathematics, "Simion Stoillow" of Romanian Academy, Bucharest 70700, Romania

e-mail: Gelu.Pasa@imar.ro 
Lake (1989) in which role of viscous instabilities on enhanced oil recovery is discussed in a practical way.

Such porous media flows enjoy some similarities with flows in a Hele-Shaw cell: in particular, one of the governing equations in both flows is Darcy's law according to which the average fluid velocity is proportional to the pressure gradient. In Hele-Shaw flows, the proportionality constant depends on the square of the thickness of the cell whereas in porous media flows, this constant is characterized by a global index, $K$, known as the permeability of the medium.

The planar material interface between two immiscible viscous fluids in a Hele-Shaw cell is well known to be unstable when a less viscous fluid pushes a more viscous fluid (Saffman and Taylor 1958) at a constant speed. The linear stability analysis shows that all modes are unstable in the absence of surface tension. Interfacial surface tension stabilizes the short waves without smearing the interface but the interface essentially remains unstable due to unstable long waves. This viscosity-driven instability is known as the Saffman-Taylor instability. Since early nineteen fifties (Chouke et al. 1959; Saffman and Taylor 1958), there has been a considerable study on this instability with and without surface tension effect on the planar interface. The review article of Saffman (1986) is worth mentioning in this connection.

An analogous problem arises in porous media flows of two immiscible fluids. In a porous medium, there is a complex network of tiny pores embedded in a solid matrix, i.e., the reservoir rock. The distribution of various fluids in the reservoir rock is greatly influenced by capillary forces during all recovery phases. On a microscopic scale, capillary forces are important in determining the amount of trapped or residual oil during displacement process. This makes capillary pressure one of the most basic rock-fluid characteristics in multi-phase flow, just as porosity and permeability are the most basic properties in single-phase flow. Although the capillary pressure in most hydrocarbon rocks may not be large, knowledge of the effects of capillary forces is extremely important for understanding fluid displacement in these rocks (Longeron et al. 1989). During displacement process of these fluids through these pores in reservoir rocks, many of these pores, if not all, contain both the immiscible phases with infinitesimal size interfaces between these fluids. Thus, there are usually many interfaces which are disjoint and disordered at microscopic level. At a macroscopic continuum level, this flow can be modeled by allowing the possibility of both the phases to co-exist at every pore. This model is truly a two-phase model, known as the saturation model which clearly differs from the Hele-Shaw model, which is essentially a one-phase model since fluids do not mix either at microscopic or macroscopic description. The saturation model, as opposed to Hele-Shaw model, includes one more equation called Buckley-Leverett equation and is widely used in modeling of immiscible flows in porous medium. Extensive literature is available on Hele-Shaw and saturation models (see (Daripa et al. 1988; Pearson 1977; Yortsos and Hickernell 1989)).

For simplicity, consider that the porous medium is initially saturated with one viscous fluid (e.g. oil). When another less viscous fluid (e.g. water) is injected in the medium, a saturation front develops across which there is a finite jump in saturation (saturation is a macroscopic concept defined as the fraction of water in water-oil mixture in the porous matrix) in the absence of capillary pressure. Unlike the material interface in Hele-Shaw flows, this saturation front is a shock wave and moves through the fluid ahead with rarefaction waves behind the shock front for a non-convex flux function. It is well known that this saturation front is also linearly unstable to all modes of perturbation. In the presence of capillary pressure which relates the pressures in two fluid phases (Dullien 1992) and is a decreasing function of the wetting phase (water here) saturation, the structure of the saturation equation changes from hyperbolic to parabolic. Physically, what this means is that capillary pressure smears out 
the sharp saturation front and thus a uniformly moving sharp saturation front is no more a possible steady state solution of the underlying equations. Instead, governing equations admit a uniformly moving smooth traveling wave as a solution whose structure depends crucially on the relation between capillary pressure and saturation. Capillary pressure is a non-linear function of saturation (see Lord et al. 1997) which makes the governing parabolic equations highly non-linear. This complicates even the linear stability analysis of such simple basic solutions. This article is about several issues related to the problem of linear stability of such traveling wave solutions arising in porous media due to capillary pressure.

On physical ground, it is easy to see that the capillary pressure has a stabilizing influence on an otherwise unstable flow in at least two different ways: (i) effect of capillary pressure in immiscible porous media flows is similar to the effect of surface tension in Hele-Shaw flows of two immiscible fluids in stabilizing the short waves; (ii) due to smooth saturation profile of the basic solution, mobility changes gradually (in space) from that of oil to that of water which has a drastic stabilizing influence on otherwise unstable waves as is well known in the context of single layer Hele-Shaw flows with smoothly varying viscosity profile (see Daripa and Hwang Submitted). The stabilizing effect of variable viscosity (mobility) has been addressed in the literature (e.g., see Pearson 1977; Jerauld et al. 1984; Huang et al. 1984). Such two-prong stabilizing influence of capillary pressure is desirable, specially in the context of enhancing oil recovery (see Daripa et al. 1988). However, conditions under which such capillary slowdown of instability can be enhanced are desirable in many applications. Such conditions, if they exist, may not be easy to find. One of the goals in this article is to find such conditions, if any and in the process investigate the control of instabilities by capillarity. In order to do so, we revisit the problem of capillary slowdown of instabilities in immiscible porous media flows. It should be mentioned here that slowing down instabilities implies that it slows down the development of fingers that result at late times. It is in this sense, the "slowdown of the viscous fingering" is synonymous with the "slowdown of growth of instabilities" for the purpose of this article.

In this article, we consider the saturation model in the presence of capillary pressure and study linear stability of traveling wave solutions. This problem has been studied by Yortsos and Hickernell (1989). Our approach here extends and simplify the analysis given there. Additionally, here we provide characterization of the almost stable modes. The results and approach here complement that of Yortsos and Hickernell (1989) in several respects which are as follows.

- First of all, we should stress the simplicity of the approach presented here which allows some characterization of profiles of stable and unstable modes.

- In Yortsos and Hickernell (1989), the stability analysis freezes the saturation-dependent coefficients in the stability equations at the values for the basic solution. In our study here, we do not freeze the coefficients. We have two new terms in the general stability system (37)-(38). The terms that contain the derivatives with respect to $S$ of the coefficients $a, b, c$, and the derivatives with respect to $x$ of the basic solution were not considered by Yortsos and Hickernell (1989) in their analysis.

- In Yortsos and Hickernell (1989), the linear stability analysis involves derivation of a differential relation between $s$ and $p$ (the amplitudes of the perturbations of water saturation and oil pressure), and a subsequent estimate of the Green's function of this differential "operator." Our approach here does not require such a differential relation between $s$ and $p$. In our approach, the system of equations for $s$ and $p$ appears as only one equation containing $s$ due to some manipulation which simplifies the stability analysis. 
- In Yortsos and Hickernell (1989), dispersion relation predicts the possibility of a most dangerous wave number for which the wave could be unstable provided initial amplitude of the wave disturbance has a local maximum far upstream in the frame moving with the traveling wave. Our result predicts that the traveling wave solution is always stable to not only short waves but also to a large class of modal perturbations having same local property as above but far downstream.

The layout of the article is as follows. In Sect. 2, we describe the saturation model and its traveling wave solutions. In Sect. 3, capillary slowdown of instabilities is shown using linear stability analysis and arguments leading to profile characterization of stable and unstable modes. Finally, we conclude in Sect. 4.

\section{Preliminaries}

\subsection{The Saturation Model}

An infinite homogeneous porous medium is considered in the plane. The medium is saturated with two immiscible phases: water and oil. The pertinent equations for this porous media flow, called saturation model here as opposed to the Hele-Shaw model, are Buckley-Leverett equation, Darcy's law, and incompressibility condition. These are given by

$$
\begin{gathered}
\phi \partial S_{i} / \partial \tau+\nabla \cdot \mathbf{v}_{i}=0, \\
\mathbf{v}_{i}=-K \lambda_{i} \nabla P_{i}, \\
P_{o}-P_{w}=P_{c}, \quad S_{o}+S_{w}=1 .
\end{gathered}
$$

We consider that phase densities and porosity are constant. Then following "incompressibility condition" results from (1) to (3) 2 .

$$
\nabla \cdot \mathbf{v}_{T}=0
$$

In Eqs. 1-4, $\tau$ refers to time, the subscripts $w$ and $o$ denote displacing (water) and displaced (oil) phases, respectively, and $\phi$ denotes the porosity of the porous medium which will be taken to be a constant. Time is denoted by $\tau$ and $\nabla$ is the gradient operator. The function $\lambda_{i}$, known as the mobility of phase $i$, is defined as $\lambda_{i}=k_{i} / \mu_{i}$, and the total velocity $\mathbf{v}_{T}=\mathbf{v}_{o}+\mathbf{v}_{w}$. The variables $S_{i}, \mathbf{v}_{i}, k_{i}, \mu_{i}$ are, respectively, saturation (volume fraction of the pore space occupied by phase $i$ ), fluid velocity, relative permeability, and viscosity of the phase $i$. The pressure in the phase $i$ is $P_{i}$, and ' $K$ ' in (2) stands for the porous medium permeability (also known as the rock permeability).

The capillary pressure $P_{c}$ in (3) is a basic parameter in the study of porous media flows containing two or more immiscible fluid phases. It relates the pressures in two fluid phases (Dullien 1992).

It is an increasing function of the non-wetting phase saturation or, alternately, a decreasing function of the wetting phase (water here) saturation. Two-phase flow models of subsurface transport often require constitutive relationship of capillary pressure as a function of saturation (Lord et al. 1997). Leverett defined a reduced capillary function containing parameters such as permeability, porosity, contact angle, surface tension, and used for correlating capillary pressure data (Dullien 1992). For modeling and correlation purposes the capillary pressure is usually described by a dimensionless so called Leverett-J function: 


$$
J\left(S_{w}\right)=\frac{P_{c}\left(S_{w}\right)}{\gamma_{o w}} \sqrt{\frac{K}{\phi}},
$$

where $\gamma_{o w}$ is the interfacial tension between the two phases. The relation (5) can be arrived at through dimensional consideration (see Stegemeier 1977). More elaborate descriptions on the capillary pressure and various constitutive relations of capillary pressure can be found elsewhere (Collins 1959; Dias and Payatakes 1986a,b; Heiba et al. 1982, 1983; Payatakes 1982).

Below, we use notations $S=S_{w}, P=P_{o}$ (pressure in the displaced phase (oil)) and the following coefficients:

$$
a(S)=K \lambda_{T}(S)>0, \quad c(S)=K \lambda_{0}(S)>0, \quad b(S)=K \lambda_{w}(S) \frac{\mathrm{d} P_{c}(S)}{\mathrm{d} S}<0,
$$

where $\lambda_{T}(S)=\lambda_{o}(S)+\lambda_{w}(S)$ is the total mobility function of saturation $S$. The Eqs. 1-4 yield the following system.

$$
\begin{aligned}
\phi \frac{\partial S}{\partial \tau}+\nabla \cdot(c(S) \nabla P) & =0, \\
\nabla \cdot(a(S) \nabla P-b(S) \nabla S) & =0 .
\end{aligned}
$$

Summing Darcy's law (2) for each phase and using the definition (3) $)_{1}$ for capillary pressure and that of total velocity $\left(\mathbf{v}_{T}=\mathbf{v}_{o}+\mathbf{v}_{w}\right)$ gives

$$
\mathbf{v}_{w}=\mathbf{v}_{T} f_{w}+c(S) f_{w}(S) P_{c}^{\prime}(S) \nabla S,
$$

where $P_{c}^{\prime}(S)$ refers to the derivative of $P_{c}(S)$ with respect to $S$, and the function $f_{w}(S)$ is defined as

$$
f_{w}(S)=\frac{\lambda_{w}(S)}{\lambda_{T}(S)} .
$$

Note that this function is equal to the ratio $v_{w} / v_{T}$ in the absence of capillary pressure. Since this ratio of velocity is usually called fractional flow function (also flux function), below we use this same name for the above function $f_{w}(S)$. Now, it follows from (1) and (9) that

$$
\phi \frac{\partial S}{\partial \tau}+\nabla \cdot\left(\mathbf{v}_{T} f_{w}+c(S) f_{w}(S) P_{c}^{\prime}(S) \nabla S\right)=0 .
$$

Above Eq. 11 is equivalent to the system (7)-(8), but does not involve pressure $P$.

Next, we discuss basic solutions (traveling waves) of the above equation whose stability to arbitrary two-dimensional perturbations is studied using (7)-(8) in Sect. 3.

\subsection{Traveling Waves}

We are interested in smooth traveling wave solutions of (11) having profile (Fig. 2)

$$
S(x, y, \tau)=\left\{\begin{array}{l}
S_{r}, x \rightarrow \infty \\
S_{l}, x \rightarrow-\infty
\end{array}\right.
$$

where $S_{l}>S_{r}$ and all derivatives of $S$ go to zero as $x \rightarrow \pm \infty$ for all $\tau \geq 0$. We see in (12) that this two dimensional basic solution of (11) does not depend on coordinate $y$. Since, the profile (12) (see also Fig. 2) is independent of $y$ (meaning it is same at every $y$ ) and hence depends only on one independent variable such as $x$ here, it is customary in the literature to refer to the basic solution (12) as one-dimensional traveling wave solution without any ambiguity. 
Before characterizing the profile of the wave, we mention for clarity that growth of instabilities at the front (i.e., the region of fast transition from left state to right state, see Fig. 2) of the traveling wave eventually gives rise to diffusive fingers, i.e., two-dimensional thin regions bounded by curves having approximate shape of "fingers." In the absence of capillary effect, these fingers have zero width in the direction of flow with effective dimension one and discontinuity in saturation across these fingers. Capillary pressure not only adds another dimension (width) to the front by smoothing the discontinuity in saturation across it, but also reduces the growth rate of instabilities at the front. This implies slowdown in the development of viscous fingers that eventually ensue from the growth of these disturbances. Next we characterize the profile of the wave connecting left state $S_{l}$ and right state $S_{r}$.

In order to implicitly construct the profile of this one-dimensional traveling wave which is useful for later purposes, we write Eq. 11 for one-dimensional flow (in the direction $x$ ) as the following evolution equation for saturation $S$.

$$
\phi \frac{\partial S}{\partial \tau}+\frac{\partial}{\partial x}\left(v_{T} f_{w}\right)=\frac{\partial}{\partial x}\left(-c(S) f_{w}(S) \frac{\mathrm{d} P_{c}}{\mathrm{~d} S} \frac{\partial S}{\partial x}\right) .
$$

Here, we have used $v_{T}$ for $\mathbf{v}_{T}$ since velocity is a scalar field now which takes a constant value for all values of $x$ (see Eq. 4). Note that since $c(S), f_{w}(S)$ are positive and $\mathrm{d} P_{c} / \mathrm{d} S$ is negative for all values of $S$, the term $\left(-c(S) f_{w}(S) \mathrm{d} P_{c} / \mathrm{d} S\right)$ in (13) is always positive.

In a moving frame $\chi=(x-U \tau) \& t=\tau$ then, the sought after traveling wave is a steady profile $\bar{S}(\chi)$ such that

$$
\bar{S}(\chi) \rightarrow S_{r} \text { as } \chi \rightarrow \infty, \quad \bar{S}(\chi) \rightarrow S_{l}>S_{r} \text { as } \chi \rightarrow-\infty, \quad \frac{\mathrm{d} \bar{S}}{\mathrm{~d} \chi} \rightarrow 0 \text { as } \chi \rightarrow \mp \infty .
$$

The speed $U$ of the traveling wave (and also the moving frame) is a constant still to be determined, and left and right states $S_{l}$ and $S_{r}$ are both constants. If $S_{r}$ is the residual value of the water saturation

$$
\frac{\mathrm{d} k_{w}}{\mathrm{~d} S}\left(S_{r}\right)=0, \quad k_{w}\left(S_{r}\right)=0
$$

Since

$$
\frac{\partial}{\partial \tau}=\frac{\partial}{\partial t}-U \frac{d}{\mathrm{~d} \chi}, \frac{\partial}{\partial x}=\frac{d}{\mathrm{~d} \chi}
$$

we have from (13) and (16),

$$
-\frac{\mathrm{d}(U \phi \bar{S})}{\mathrm{d} \chi}+\frac{d}{\mathrm{~d} \chi}\left(v_{T} f_{w}\right)=-\frac{d}{\mathrm{~d} \chi}\left(c(\bar{S}) f_{w}(\bar{S}) \frac{\mathrm{d} P_{c}}{\mathrm{~d} S} \frac{\mathrm{d} \bar{S}}{\mathrm{~d} \chi}\right) .
$$

Integrating once, we obtain

$$
v_{T} f_{w}-U \phi \bar{S}+c(\bar{S}) f_{w}(\bar{S}) \frac{\mathrm{d} P_{c}}{\mathrm{~d} S} \frac{\mathrm{d} \bar{S}}{\mathrm{~d} \chi}=A,
$$

where $A$ is a constant of integration. The relation (9) for the water phase in one-dimensional flow and the relation (18) give

$$
A=v_{w}-U \phi \bar{S}
$$

where $v_{w}$ is the speed of the water phase. Relation (18) suggests that if a steady profile $\bar{S}(\chi)$ satisfying (14) exists, then the free parameters $U$ and $A$ must satisfy

$$
-U \phi S_{l}+v_{T} f_{w}\left(S_{l}\right)=-U \phi S_{r}+v_{T} f_{w}\left(S_{r}\right)=A,
$$


Fig. 1 A typical flux function $f_{w}(s)$ given by (10) and the line (D) given by (23) are shown

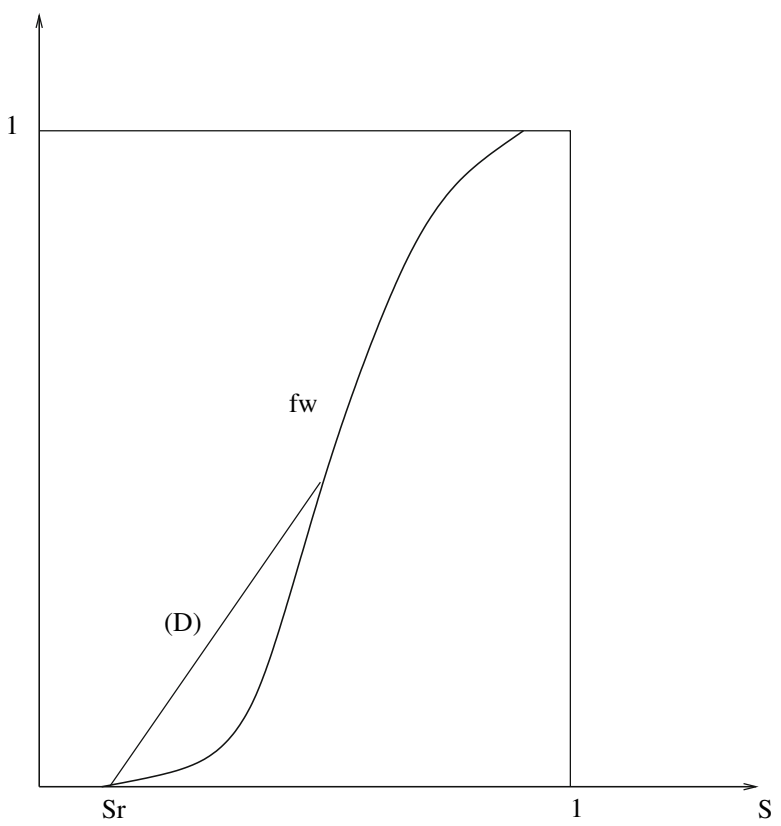

from which we obtain

$$
U=\frac{v_{T}}{\phi}\left(\frac{f_{w}\left(S_{l}\right)-f_{w}\left(S_{r}\right)}{S_{l}-S_{r}}\right)
$$

This relation between the speed of the traveling wave and the two states at infinity is expected from well established theory on nonlinear waves.

An implicit relation for the basic solution $\bar{S}(\chi)$ is obtained from (18) in the form

$$
\chi-\chi_{o}=\int_{\bar{S}(0)}^{\bar{S}} \frac{\psi(S) \mathrm{d} S}{(A+U \phi \bar{S})-v_{T} f_{w}}, \quad \psi(S)=c(S) f_{w}(S) \frac{\mathrm{d} P_{c}}{\mathrm{~d} S}<0,
$$

where $\bar{S}(0)=\bar{S}\left(\chi_{0}\right)$. Clearly, behavior of the integral (22) is given by the set of intersection points of the line $(D)$ (see Fig. 1) given by

$$
l(S)=(A+U \phi S) / v_{T},
$$

with the fractional flow curve $f_{w}(S)$ defined by (10). Note that the slope of the line $l(S)$ defined by Eq. 23 is $U \phi / v_{T}$ which, according to Eq. 21, is the slope of the chord connecting the points $f_{w}\left(S_{r}\right)$ and $f_{w}\left(S_{l}\right)$ on the graph of the flux function $f_{w}(S)$. This is illustrated in Fig. 1. Therefore, the denominator of the kernel in the integral is positive for all values of $S$ in the interval $\left(S_{r}, S_{l}\right)$ (Recall from (14) that $\left.S_{l}>S_{r}\right)$ so long as these states $S_{l}$ and $S_{r}$ are on the convex part of the flux function as shown in the figure. Therefore, the sign of the kernel in the integral is negative (since $\Psi(S)<0$ ) for all values of $S$ in the interval $\left(S_{r}, S_{l}\right)$. Hence, $\chi$ is a monotonically decreasing function of $\bar{S}$ for any $\bar{S} \in\left[S_{r}, S_{l}\right]$, Upon inversion, we see that traveling wave saturation profile $\bar{S}(\chi)$ is a monotonically decreasing function of $\chi$ for $S_{l}>S_{r}$ as shown in Fig. 2.

We denote by $\bar{P}$ the basic state of oil pressure, corresponding to $\bar{S}$ given implicitly by (22). We also look for a steady profile $\bar{P}$ in the moving coordinate $\chi$. Then Eq. 7 for one-dimensional flow in the moving coordinate $\chi$ becomes 


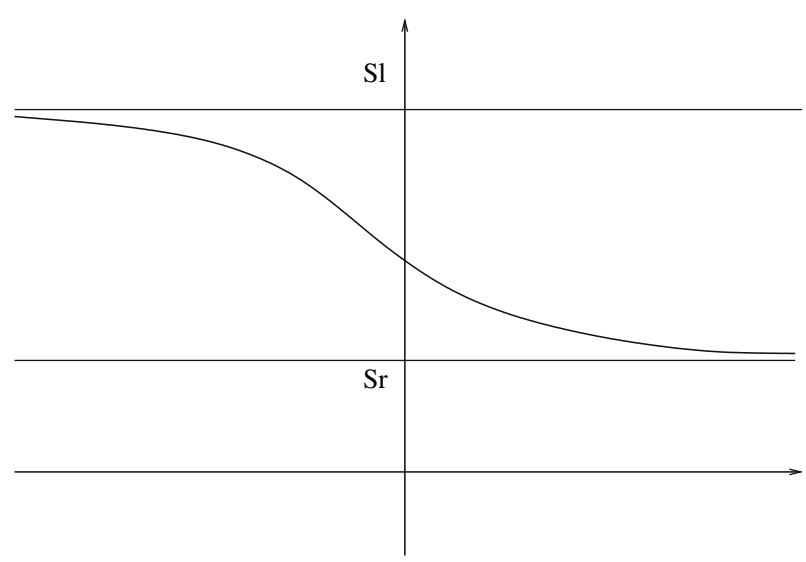

Fig. 2 A typical traveling wave saturation profile in the moving frame is shown

$$
-U \phi \frac{\mathrm{d} \bar{S}}{\mathrm{~d} \chi}+\frac{d}{\mathrm{~d} \chi}\left(c(\bar{S}) \frac{\mathrm{d} \bar{P}}{\mathrm{~d} \chi}\right)=0
$$

Therefore the basic solution profiles $(\bar{S}, \bar{P})$ as traveling waves are solutions of the system

$$
\begin{aligned}
c(\bar{S}) f_{w}(\bar{S}) \frac{\mathrm{d} P_{c}}{\mathrm{~d} S} \frac{\mathrm{d} \bar{S}}{\mathrm{~d} \chi} & =U \phi \bar{S}-v_{T} f_{w}(\bar{S})+A, \\
c(\bar{S}) \frac{\mathrm{d} \bar{P}}{\mathrm{~d} \chi} & =U \phi \bar{S}+C,
\end{aligned}
$$

where $C$ is a constant. The second of these equations, namely (26), is obtained from integrating (24). Using (19) and (25) we see that $\frac{\mathrm{d} \bar{S}}{\mathrm{~d} \chi} \rightarrow 0$ when $\bar{S} \rightarrow S_{r}$.

For the purpose of our analysis in sections below, we do not need to know the pressure and saturation solutions of the above equations. Actually, we have already discussed qualitative behavior of the saturation solution in the paragraph immediately following Eq. 23. Solving for the pressure solution of the above equations is no less difficult than similar problem that arises in the context of Navier-Stokes (NS) equations. In our case here, the problem for the saturation is a Cauchy problem (see Eq. 7) whereas the problem for the pressure is governed by a Poisson-type equation (see Eq. 8) for which the boundary data (far-field) data is usually not provided and need to be estimated from the Cauchy-problem for saturation, analogous to the case for Navier-Stokes' equation. Nonetheless, for the sake of completeness, we outline a procedure to obtain this pressure solution in terms of two constants. These two constants, of course, can be found if boundary conditions for the pressure can be estimated.

Note that Eq. 26 can be rewritten as

$$
c(\bar{S}) \frac{\mathrm{d} \bar{S}}{\mathrm{~d} \chi} \frac{d \bar{P}}{d \bar{S}}=U \phi \bar{S}+C .
$$

Equations 18 and 20 can be used to obtain, after some simplification, the following equation.

$$
c(\bar{S}) \frac{\mathrm{d} \bar{S}}{\mathrm{~d} \chi} f_{w}(\bar{S}) \frac{\mathrm{d} P_{c}}{\mathrm{~d} S}=v_{T}\left(S_{r}-\bar{S}\right)\left(\frac{f_{w}\left(S_{r}\right)-f_{w}(\bar{S})}{S_{r}-\bar{S}}-\frac{[f]}{[S]}\right) \equiv g(\bar{S})>0,
$$


where $[f]=\left(f_{w}\left(S_{r}\right)-f_{w}\left(S_{l}\right)\right)$ and $[S]=\left(S_{r}-S_{l}\right)$. Note that (28) also defines the function $g(\bar{S})$ which is greater than zero for $S_{r}<\bar{S}<S_{l}$. Then from Eqs. 27 and 28, we can write

$$
\frac{\mathrm{d} \bar{P}}{\mathrm{~d} \bar{S}}=f_{w}(\bar{S}) \frac{\mathrm{d} P_{c}}{\mathrm{~d} S}(U \phi \bar{S}+C) / g(S), \quad S_{r} \leq \bar{S} \leq S_{l} .
$$

Note that there is already an unknown constant $C$ in this equation and right hand side is only a function of $\bar{S}$ keeping in mind that $\frac{\mathrm{d} P_{c}}{\mathrm{~d} S}$ (which is a function of $\bar{S}$ ) is a negative quantity usually for a wetting phase such as water in our case. Integrating this Eq. 29, we can find $\bar{P}(\bar{S})$ in terms of two constants to be determined by two boundary conditions on $\bar{P}$. However, we do not see any need to further continue with this profile of $\bar{P}(\bar{S})$ since this falls outside the scope of this article and in any case, is not needed below in our analysis.

\section{Capillary Slowdown via Linear Stability Analysis}

Consider perturbations of traveling wave solutions, $(\bar{S}, \bar{P})$, of the system (25)-(26):

$$
S(x, y, t)=\bar{S}(\chi)+\epsilon s_{1}, \quad P(x, y, t)=\bar{P}(\chi)+\epsilon p_{1} .
$$

where $\epsilon$ is a small parameter and

$$
s_{1}, p_{1} \rightarrow 0, \text { as } x \rightarrow \mp \infty,
$$

and also all its derivatives go to zero as $x \rightarrow \mp \infty$ for each $y$ and $t \geq 0$. We expand the functions $a, b, c$ defined in (6) in Taylor series about $\bar{S}$ to obtain expressions of the form

$$
a\left(\bar{S}+\epsilon s_{1}\right)=a(\bar{S})+a_{S}(\bar{S}) \epsilon s_{1}+O\left(\epsilon^{2}\right)
$$

for $a, b, c$. Here the subscript $s$ denotes derivative with respect to $\bar{S}$.

We transform (7) and (8) in a moving frame $\chi=x-U t$, in which we first substitute (30) and (32) and then equate to zero the coefficients of like powers in the small parameter $\epsilon$. At order $O(\epsilon)$, we obtain the following linearized equations for $s_{1}$ and $p_{1}$.

$$
\begin{aligned}
& \phi \frac{\partial s_{1}}{\partial t}-\phi U \frac{\partial s_{1}}{\partial \chi}+\nabla \cdot\left(c(\bar{S}) \nabla p_{1}+c_{s} s_{1} \nabla \bar{P}\right)=0, \\
& \nabla \cdot\left(a(\bar{S}) \nabla p_{1}+a_{s} s_{1} \nabla \bar{P}-b(\bar{S}) \nabla s_{1}-b_{s} s_{1} \nabla \bar{S}\right)=0 .
\end{aligned}
$$

We study the temporal evolution of arbitrary perturbations by the method of normal modes. Hence, we consider a typical wave component of the form

$$
\left(s_{1}, p_{1}\right)=(s(\chi), p(\chi)) e^{(i k y+\sigma t)},
$$

where $k$ is a real axial wavenumber and $\sigma$ is the growth rate. From (30) and (35), we obtain

$$
s(\chi) \rightarrow 0 \text { as } \chi \rightarrow \mp \infty, \quad \frac{\mathrm{d} s}{\mathrm{~d} \chi} \rightarrow 0 \text { as } \chi \rightarrow \mp \infty .
$$


We substitute (35) into (33) and (34), and obtain

$$
\begin{gathered}
p^{\prime \prime} \bar{c}+p^{\prime} c^{\prime}(\bar{S})-\bar{c} k^{2} p+\sigma \phi s-\phi U s^{\prime}+\frac{d}{\mathrm{~d} \chi}\left(c_{S}(\bar{S}) s \frac{\mathrm{d} \bar{P}}{\mathrm{~d} \chi}\right)=0, \\
p^{\prime \prime} \bar{a}+p^{\prime} a^{\prime}(\bar{S})-\bar{a} k^{2} p-s^{\prime \prime} \bar{b}-b^{\prime}(\bar{S}) s^{\prime}+\bar{b} k^{2} s \\
+\frac{d}{\mathrm{~d} \chi}\left(a_{s}(\bar{S}) \frac{\mathrm{d} \bar{P}}{\mathrm{~d} \chi} s-b_{s}(\bar{S}) \frac{\mathrm{d} \bar{S}}{\mathrm{~d} \chi} s\right)=0,
\end{gathered}
$$

where $^{\prime}=d / d \chi$ and $\bar{a}, \bar{b}, \bar{c}$ are the values of $a, b, c$ for the traveling wave $\bar{S}$. The system (37)-(38) holds in the domain $\chi \in(-\infty, \infty)$ of the porous medium. Terms in the above system containing $a_{S}(\bar{S}), b_{S}(\bar{S}), c_{S}(\bar{S})$ and the partial derivatives of $\bar{S}$ and $\bar{P}$ do not appear in the corresponding system studied in Yortsos and Hickernell (1989).

Below, we give an analysis of the normal modes in the far-field for the system of Eqs. 37 and 38. As we will see, this leads to a system of equations (see (41) and (42) below) which still depend on the basic traveling wave profile but an estimate of the growth rate based on these equations do not. Therefore, we believe that conclusions about the growth rates based on far-field analysis are likely to hold also at the front, although further research is required to strengthen the validity of this statement.

Equations 37 and 38 simplify in the far field which are easier to analyze. In order to see the simplification, first note from Eq. 26 that $\bar{P}^{\prime}$ approaches a constant value, say $\bar{P}^{\prime}{ }_{\infty}$, far downstream and we know that $\bar{S}(\chi) \rightarrow S_{r}$ as $\chi \rightarrow \infty$. Then for a given $\epsilon>0$ however small, there exists a point $R$ (large enough) such that $\left|\bar{S}(\chi)-S_{r}\right|,\left|\bar{P}^{\prime}(\chi)-\bar{P}_{\infty}^{\prime}\right|<\epsilon$ for $\chi>R$. These differences are decreasing functions of the distance of the point $R$. We choose a value $R$ so far downstream that these differences are small enough to have any effect in our leading order Eqs. 37 and 38 in the far field $\chi>R$. Therefore, we can set $\bar{S}(\chi)=S_{r}$, and $\bar{P}^{\prime}(\chi)=$ constant for $\chi>R$ in (37) and (38). Note that this is also consistent physically in an infinite porous media with $S_{r}$ as the residual saturation, because wave can travel only so far to have no effect beyond the distance it has traveled. We analyze now the terms of (37)-(38) in the far field. We can write the last term of Eq. 37 as

$$
\frac{d}{\mathrm{~d} \chi}\left(c_{s}(\bar{S}) s \frac{\mathrm{d} \bar{P}}{\mathrm{~d} \chi}\right)=\frac{\mathrm{d}^{2} c(\bar{S})}{\mathrm{d} \bar{S}^{2}} \frac{\mathrm{d} \bar{S}}{\mathrm{~d} \chi} \frac{\mathrm{d} \bar{P}}{\mathrm{~d} \chi} s+c_{s}(\bar{S}) \frac{\mathrm{d}^{2} \bar{P}}{\mathrm{~d} \chi^{2}} s+s^{\prime} c_{s}(\bar{S}) \frac{\mathrm{d} \bar{P}}{\mathrm{~d} \chi} \approx s^{\prime} c_{s}(\bar{S}) \frac{\mathrm{d} \bar{P}}{\mathrm{~d} \chi}, \quad \chi \geq R,
$$

A similar expression holds for the term containing the coefficient $a_{s}$ in (38). It is worth noting from (9) that in the far-field, where $\nabla \bar{S}=0$, we have $\mathbf{v}_{w}=\mathbf{v}_{T} f_{w}$ and from (19) that the right hand side of (25) is zero in the far-field.

We consider next the system (37), (38) \& (39) in the domain $\chi \geq R$. Therefore the terms containing the derivatives of $\bar{S}$ and $\bar{P}^{\prime}$ with respect to $\chi$ are equal to zero. Moreover, we have

$$
\bar{a}^{\prime}(\chi)=a^{\prime}(\bar{S}(\chi))=\frac{\mathrm{d} \bar{a}}{\mathrm{~d} \chi}(\bar{S})=\frac{\mathrm{d} a}{\mathrm{~d} S}(\bar{S}) \frac{\mathrm{d} \bar{S}}{\mathrm{~d} \chi}=0, \quad \forall \chi \geq R,
$$


and analogous formulae for $\bar{b}^{\prime}$ and $\bar{c}^{\prime}$. Then in the region $\chi \geq R, \bar{a}^{\prime}=\bar{b}^{\prime}=\bar{c}^{\prime}=0$. and the system (37)-(38) reduces to

$$
\begin{aligned}
& p^{\prime \prime} c\left(S_{r}\right)-k^{2} p c\left(S_{r}\right)+\phi \sigma s-\phi U s^{\prime}+s^{\prime} c_{s}\left(S_{r}\right) \frac{\mathrm{d} \bar{P}}{\mathrm{~d} \chi}=0, \quad \forall \chi \geq R, \\
& p^{\prime \prime} a\left(S_{r}\right)-k^{2} p a\left(S_{r}\right)-s^{\prime \prime} b\left(S_{r}\right)+k^{2} s b\left(S_{r}\right)+s^{\prime} a_{s}\left(S_{r}\right) \frac{\mathrm{d} \bar{P}}{\mathrm{~d} \chi}=0, \quad \forall \chi \geq R .
\end{aligned}
$$

We multiply the first equation by $a\left(S_{r}\right)$ and the second equation by $c\left(S_{r}\right)$. We subtract and obtain only one relation for the unknown eigenfunction $s(\chi)$.

$$
\begin{aligned}
& b\left(S_{r}\right) c\left(S_{r}\right)\left(s^{\prime \prime}-k^{2} s\right)+\sigma \phi a\left(S_{r}\right) s-U \phi a\left(S_{r}\right) s^{\prime} \\
& +s^{\prime} \frac{\mathrm{d} \bar{P}}{\mathrm{~d} \chi}\left(a\left(S_{r}\right) c_{s}\left(S_{r}\right)-c\left(S_{r}\right) a_{s}\left(S_{r}\right)\right)=0, \quad \forall \chi \geq R .
\end{aligned}
$$

We use the relations (6) and (15) and obtain:

$$
\begin{aligned}
\left.a\left(S_{r}\right) c_{s}\left(S_{r}\right)\right)-c\left(S_{r}\right) a_{S}\left(S_{r}\right) & =\frac{K^{2}}{\mu_{w} \mu_{o}}\left(k_{w}\left(S_{r}\right) \frac{\mathrm{d} k_{o}}{\mathrm{~d} S}\left(S_{r}\right)-k_{o}\left(S_{r}\right) \frac{\mathrm{d} k_{w}}{\mathrm{~d} S}\left(S_{r}\right)\right) \\
& =0, \quad \forall \chi \geq R .
\end{aligned}
$$

From (43) and (44), we have

$$
b\left(S_{r}\right) c\left(S_{r}\right)\left(s^{\prime \prime}-k^{2} s\right)-a\left(S_{r}\right) \phi U s^{\prime}+\sigma \phi a\left(S_{r}\right) s=0 .
$$

The general solution $S$ of (7)-(8) and the basic solution $\bar{S}$ of (25) satisfy the same boundary condition at $x=\infty$. Hence we have $s(\infty)=0$ which together with Eq. (45) constitute the following eigenvalue problem:

$$
\begin{aligned}
-\alpha s^{\prime \prime}+\phi U s^{\prime}+\alpha k^{2} s & =\omega s, \quad \chi>R, \\
s(\infty) & =0,
\end{aligned}
$$

where

$$
\alpha=b\left(S_{r}\right) c\left(S_{r}\right) / a\left(S_{r}\right)<0, \quad \omega=\sigma \phi, \phi>0 .
$$

Theorem 1 The growth rate $\sigma$ is real. Therefore, no modes get convected and stability (or instability) is purely absolute in nature.

Proof Since $s_{1}$ (see (30)) is real, the ansatz (35) implies that

$$
s(\chi ; k)=s^{*}(\chi ;-k)
$$

where a “ $*$ " stands for complex conjugate. Note from (46) that $s$ also depends on the wavenumber $k$. In the ansatz (35), the dependence of $s$ on wavenumber $k$ is not explicitly expressed, though implied. Using the above relation in the equation resulting from taking the complex conjugate of the Eq. 46, we obtain

$$
-\alpha s^{\prime \prime}+\phi U s^{\prime}+\alpha k^{2} s=\omega^{*} s, \quad \chi>R .
$$

So, it follows from (46) and (50) that $\omega=\omega^{*}$ meaning the growth rate is real. 
Theorem 2 The eigenfunction " $s$ " of Eq. (46) is real

Proof Since perturbations are real, Fourier series expansion $\sum_{k=-\infty}^{\infty} s(\chi ; k) e^{i k y}$ of the initial perturbation implies that $s(\chi ; k)$ satisfies (49). Since $k$ in Eq. 46 appears as $k^{2}$ only, then solution of this equation must satisfy

$$
s(\chi ; k)=s(\chi ;-k) .
$$

These two relations (49) and (51) show that eigenfunction $s(\chi ; k)$ is real.

Consider a point $M>R$ at a finite distance far downstream. We multiply the relation (46) by $s$, and integrate over the interval $[M, \infty)$. Then we obtain

$$
\begin{array}{r}
\alpha\left\{\int_{M}^{\infty}\left(s^{\prime 2}+k^{2} s^{2}\right) \mathrm{d} \chi-s^{\prime}(\infty) s(\infty)+s^{\prime}(M) s(M)\right\}+\phi U\left\{s^{2}(\infty)-s^{2}(M)\right\} / 2 \\
=\omega \int_{M}^{\infty} s^{2} \mathrm{~d} \chi
\end{array}
$$

Using (47) we get

$$
\alpha\left\{\int_{M}^{\infty}\left(s^{\prime 2}+k^{2} s^{2}\right) \mathrm{d} \chi+s^{\prime}(M) s(M)\right\}-\frac{\phi}{2} U s^{2}(M)=\omega \int_{M}^{\infty} s^{2} \mathrm{~d} \chi,
$$

and therefore the growth rate $\sigma$ is given by

$$
\begin{aligned}
\sigma & =\alpha \frac{\left\{\int_{M}^{\infty}\left(s^{\prime 2}+k^{2} s^{2}\right) \mathrm{d} \chi+0.5\left(s^{2}\right)^{\prime}(M)\right\}}{\phi \int_{M}^{\infty} s^{2} \mathrm{~d} \chi}-\frac{U s^{2}(M)}{2 \int_{M}^{\infty} s^{2} \mathrm{~d} \chi} \\
& <\alpha \frac{\left\{\int_{M}^{\infty}\left(s^{\prime 2}+k^{2} s^{2}\right) \mathrm{d} \chi+0.5\left(s^{2}\right)^{\prime}(M)\right\}}{\phi \int_{M}^{\infty} s^{2} \mathrm{~d} \chi}
\end{aligned}
$$

Recall from (48) that $\alpha<0$ in the above formula. Now, we draw the following inferences from the above formula.

Note that the integrals in (54) are positive. This can not be said of the only other term $\left(s^{2}\right)^{\prime}(M)$ in general for any disturbance. If this were the case, then this would imply stability in general. Even though this is not the case, the situation is very close to it in the following sense which makes the capillary slowdown very dramatic. Short waves are certainly stable because the integral in the numerator is always going to be larger than $\left|\left(s^{2}\right)^{\prime}(M)\right|$ for $k$ beyond some threshold. Moreover, this threshold value need not be very large considering the fact that the point $M>R$ in the far field can be taken to be the point where $\left|\left(s^{2}\right)^{\prime}(M)\right|$ assumes the least value. In this sense, only very long waves are possibly unstable, rest being stable and it is in this sense that the stabilizing effect of capillarity is termed as dramatic. Moreover, the class of modal perturbations for which $\left|\left(s^{2}\right)^{\prime}\right|=0$ at a point $M>R$ in the far-field are always stable. Such a stable modal perturbation only needs to have either its amplitude zero or its amplitude's local extremum at a point $M>R$.

Remark 1 At this point, it is worth mentioning some details of stability analysis given in Yortsos and Hickernell (1989). Thus, below all notations and equations refer to Yortsos and Hickernell (1989). There, amplitude of perturbation is normalized using the derivative of basic saturation at a finite point in the mobile system $\xi$. The normalized amplitude $\Phi$ is given in the definition (3.3) there as $\Phi=s /(d \bar{S} / d \xi)$. After some calculation, following stability equation (4.3) in Yortsos and Hickernell (1989) is obtained. 


$$
\frac{\omega}{v}=\left\{-\frac{\mu}{\lambda_{T}} \Phi^{\prime \prime}-\left(\frac{\mu}{\lambda_{T}}\right)^{\prime} \Phi^{\prime}+\alpha^{2} \frac{\mu}{\lambda_{T}} \Phi+\alpha^{2}\left[\zeta^{\prime}-\left(\frac{\mu}{\lambda_{T}}\right)^{\prime}\right] W\right\} / \zeta^{\prime} \Phi,
$$

where $\lambda_{T}$ is the normalized mobility, $W$ is a function of water saturation and oil pressure, $\mu=\zeta \lambda_{T}+\lambda_{0}, \zeta=v S+f_{w}^{\infty}-1, v$ is traveling velocity-see notations (3.4b)-(3.8). Here $\omega$ is the growth constant and $\alpha$ is the wave-number. The stability analysis of the above equation by the authors there implicitly assumes existence of a upstream point where $\Phi$ has a local maximum. This assumption precludes a large class of modal disturbance whose amplitude might decay monotonically away from the origin in the region $\xi<0$ (using the notation of Yortsos and Hickernell (1989)) which is the region of interest in their analysis.

We mention the following theorems followed by simple proofs.

Theorem 3 A mode whose amplitude decays with oscillations as $\chi \rightarrow \infty$ is stable.

Proof Consider a mode whose amplitude decays exponentially with oscillations in the far field $\chi \rightarrow \infty$. Then there must exist a point $M$ in the far field where $s^{2}$ attains a maximum and therefore it follows from (54) that $\sigma<0$ for such a mode (recall $\alpha<0$ in (54)).

A direct consequence of this theorem is the following corollary.

Corollary 1 Amplitude of an unstable mode decays exponentially without oscillations as $\chi \rightarrow \infty$.

In the proof above, it is clear that a mode whose amplitude decays with oscillations in the far field must be stable. However, note from (54) that $\sigma$ can be negative even if there is no point $M$ in the far field where $\left(s^{2}\right)^{\prime}$ is zero and therefore, amplitude of a stable mode can decay without oscillations in the far field. We summarize this in the following theorem.

Theorem 4 Amplitude of a stable mode can decay with or without oscillations in the far field.

We can characterize the behavior of these short (stable) and long (unstable) waves as $\chi \rightarrow \infty$ more precisely if we analyze the solution of the problem defined in (46). The Eq. 46 admits solutions of the type $\exp (r \chi)$ if $r$ satisfies the characteristic equation:

$$
r^{2}+\tilde{U} r-\left(v+k^{2}\right)=0,
$$

where $\tilde{U}=-\phi U / \alpha$ and $v=-\omega / \alpha$. Note that $\tilde{U}>0$. The roots $r_{1}$ and $r_{2}$ of this characteristic equation are given by $r_{1,2}=-\tilde{U} / 2 \pm \sqrt{(\tilde{U} / 2)^{2}+\left(v+k^{2}\right)}$ where subscripts 1 and 2 correspond to the + and - signs respectively. The following cases arise from this.

1. If $v+k^{2}>0$, then only the negative root is admissible due to the boundary condition defined in (46). Since $v+k^{2}>0$ implies growth rate $\sigma>\phi \alpha k^{2}$ (recall $\alpha<0, \phi>0$ ), we conclude that unstable long waves decay exponentially without oscillations in space as $\chi \rightarrow \infty$ for any fixed $t>0$.

2. If $-(\tilde{U} / 2)^{2}<\left(v+k^{2}\right)<0$, both the roots are negative and if $-(\tilde{U} / 2)^{2}>\left(v+k^{2}\right)$, then both the roots are complex with negative real part. Since in both of these cases $v+k^{2}<0$ which means $\sigma<\phi \alpha k^{2}$, we conclude that the stable short waves can decay exponentially with or without oscillations in space as $\chi \rightarrow \infty$ which will depend on whether $-(\tilde{U} / 2)^{2}$ is greater or less than $\left(v+k^{2}\right)$, respectively.

Note that implications by the above analysis are consistent with the Theorems 3 and 4 as it should be. 
Remark 2 In Yortsos and Hickernell (1989), a strict upper bound on the growth rate is a parabola in wavenumber $k$ with a positive maximum point and a finite cut-off wave-number beyond which the growth rate is negative. Our result in (54) can be interpreted in a somewhat similar fashion leading to stronger results for a certain class of disturbances in the far field. In order to show this, we rewrite (54) as

$$
\sigma<\left(\frac{\alpha}{\phi}\right)\left(k^{2}+\frac{\int_{M}^{\infty} s^{\prime 2} \mathrm{~d} \chi}{\int_{M}^{\infty} s^{2} \mathrm{~d} \chi}+\frac{0.5\left(s^{2}\right)^{\prime}(M)}{\int_{M}^{\infty} s^{2} \mathrm{~d} \chi}\right) .
$$

Recall that $\alpha<0$ and $\phi>0$. The leading term of the bound on the growth rate $\sigma$ is a parabola in wave-number $k$ with a negative maximum point. The second term is negative which makes the value of the upper bound even more negative except for the shift due to the term involving $\left(s^{2}\right)^{\prime}(M)$ whose sign can be determined a priori in general. If initial amplitude $s(\chi)$ of any wave disturbance in the far field has the property that $\left(s^{2}\right)^{\prime}(M) \geq 0$, then such waves are certainly stable since all terms above in (56) is negative. This result precisely characterizes the growth rate of stable waves in the far-field.

\section{Conclusions}

We have presented a simple approach to show that the traveling wave solutions of the saturation model (1) through (4) with the property (14) are stable to short wave perturbations and alluded to the fact the bandwidth of such waves is very large in general meaning the bandwidth of the unstable long waves is possibly very small. This brings out some of differences between the role of surface tension in immiscible Hele-Shaw flows and the role of capillarity in immiscible porous media flow. Surface tension and capillarity both stabilizes the short waves in respective flows, but the effect of capillarity is more severe as it tends to stabilize almost entire spectrum of waves (except the very small ones). This, in some sense, quantifies the physics-based expectation, discussed earlier in Sect. 1, of the two prong stabilizing influence of capillarity on disturbances. Another distinction is that a large class of modal perturbations, for which $\left(s^{2}\right)^{\prime}=0$ far downstream at a point $M>R$, exist which are completely stabilized by capillarity.

In summary, we mention following results obtained in this article.

1. Growth rate of perturbations, which is not in contradiction with the upper bound obtained in Yortsos and Hickernell (1989), is analyzed through weak formulation as well as exact solution of the underlying ordinary differential equation for perturbation. In Sect. 3, we establish that The short waves are stable and long ones are unstable. The width of the unstable band of long waves is infinitesimally small.

2. The instability (stability) of each unstable (stable) mode is absolute in nature. We characterize the asymptotic structure of these modes: Amplitude of an unstable mode decays (in space) exponentially without oscillations, and that of a stable mode can decay with or without oscillations as $\chi \rightarrow \infty$. It then follows that a mode whose amplitude decays with oscillations as $\chi \rightarrow \infty$ must be stable.

3. The traveling wave can be stabilized by controlling disturbance amplitude $s(\chi)$ at only one point in the far field downstream such that $\left(s^{2}\right)^{\prime}=0$ at that point.

In closing, we want to add some general comments regarding two aspects having to do with the title and content of this article: (i) the role of viscous fingering on enhanced oil recovery, and (ii) the importance of capillary pressure on stability and oil recovery. In homogeneous 
reservoir with significant vertical permeability (i.e., in the $x$ - direction for this article) and negligible gravity effect, the case considered in the article, viscous fingering is certainly very important and plays a significant role in reducing sweeping efficiency of flooding in oil recovery. Thus viscous fingering is a serious impediment to oil recovery. Hence, slowing down the development of viscous fingering by any means is desirable for enhanced oil recovery. We have shown in this article that capillary forces play such a positive effect on enhanced oil recovery by slowing down the growth of instabilities that lead to viscous fingering. This, de facto, sheds light on the second aspect mentioned above, namely, capillary pressure which is important for stability and oil recovery.

Since there are no homogeneous field reservoirs in general, flow in most field reservoirs is mostly dominated by channeling through high permeable zones. Although, viscosity driven instability is present here, it does not lead to significant viscous fingering due to heterogeneity in permeability. Thus, viscous fingering is a secondary effect for oil recovery in such heterogeneous reservoirs. It is worth mentioning here that in Daripa and Pásá (2004), it has been shown that viscosity driven instability in mildly heterogeneous reservoirs mitigates (see Daripa and Pásá 2004) to some extent the tendency of preferential channeling of flow through high permeable regions. The role of capillary pressure on oil recovery and stability is no different for heterogeneous reservoir than for homogeneous reservoir. For reasons mentioned earlier, capillary force is extremely important for fluid displacement processes through reservoir rocks and hence for oil recovery and stability.

Acknowledgements The authors sincerely thank both the referees for providing very insightful questions and addressing these questions during the revision process has significantly improved the article in our opinion. The second author is grateful to the Department of Mathematics at Texas A \& M University (USA) for hospitality during a visit in the summer of 2005 when this research was carried out. This project was partially supported by GRANT CEx06-11-12.

\section{References}

Al-Hadhrami, H.S., Blunt, M.J.: Thermally induced wettability alteration to improve oil recovery in fractured reservoirs. SPE Res. Eval. Eng. 4, 179-186 (2001)

Chouke, R.L., Van Meurs, P., Van Der Pol, L.B.: The stability of a slow, immiscible, viscous liquid-liquid displacement in a permeable media. Trans. AIME 216, 188-194 (1959)

Collins, R.E.: Flow of fluids through porous materials. AIME 216, 188-194 (1959)

Craig, F.F. Jr.: The Reservoir Engineering Aspects of Waterflooding. Monograph Series 3, SPE, Dallas (1971)

Daripa, P., Hwang, H.J.: Nonlinear Saffman-Taylor Instability for Hele-Shaw Flows (Submitted)

Daripa, P., Pásá, G.: An optimal viscosity profile in enhanced oil recovery by polymer flooding. Int. J. Eng. Sci. 42, 2029-2039 (2004)

Daripa, P., Glimm, J., Lindquist, B., McBryan, O.: Polymer floods: a case study of nonlinear wave analysis and instability control in tertiary oil recovery. SIAM J. Appl. Math. 49, 353-373 (1988)

Dias, M.M., Payatakes, A.C.: Network models for two-phase flow in porous media, Part I Immiscible microdisplacement of non-wetting fluids. J. Fluid Mech. 164, 305-336 (1986a)

Dias, M.M., Payatakes, A.C.: Network models for two-phase flow in porous media, Part II Motion of oil ganglia. J. Fluid Mech. 164, 337-358 (1986b)

Dullien, F.A.L.: Porous media fluid transport and pore structure. Academic press (1992)

Heiba, A.A., Sahimi, M., Davis, H.T., Scriven, L.E.: Percolation theory of two-phase relative permeability. Paper SPE 11015 presented at the 57th SPE Annual Technical Conference and Exhibition. New Orleans, September 26-29 (1982)

Heiba, A.A., Davis, H.T., Scriven, L.E.: Effects of wettability on two-phase relative permeabilities and capillary pressure. Paper SPE 12172 presented at the 58th SPE Annual Technical Conference and Exhibition, San Francisco, October 26-29 (1983)

Huang, A.B., Chikhliwala, E.D., Yortsos, Y.C.: Linear stability analysis of immiscible displacement: Part II general basic flow profiles. Paper SPE 13163 presented at the 59th SPE Annual Technical Conference and Exhibition, Houston, September 16-19 (1984) 
Jerauld, G.R., Davis, H.T., Scriven, L.E.: Stability fronts of permanent form in immiscible displacement. Paper SPE 13164 presented at the 59th SPE Annual Technical Conference and Exhibition, Houston, September $16-19(1984)$

Lake, L.W.: Enhanced Oil Recovery. Prentice Hall (1989)

Longeron, D.G., Argaud, M.J., Bouvier, L.: SPE-19589 (1989)

Lord, D.L., Demond, A.H., Salehzadeh, A., Hayes, K.F.: Influence of organic and solution chemistry on subsurface transport properties. 2. Capillary pressure-saturation. Environ. Sci. Technol. 31, 2052-2058 (1997)

Payatakes, A.C.: Dynamics of oil ganglia during immiscible displacement in water-wet porous media. Ann. Rev. Fluid Mech. 14, 365-393 (1982)

Pearson, H.J.: The stability of some variable viscosity flow with application in oil extraction. Cambridge University Report (1977)

Saffman, P.G.: Viscous fingering in Hele-Shaw cells. J. Fluid Mech. 173, 73-94 (1986)

Saffman, P.G., Taylor, G.I.: The penetration of a fluid in a porous medium or Hele-Shaw cell containing a more viscous fluid. Proc. Roy. Soc. A. 245, 312-329 (1958)

Stalkup, F.I.: Miscible Displacement. Monograph Series 8, SPE, Dallas (1989)

Stegemeier, G.L.: Oil entrapment and mobilization in porous media. In: Shaw, D.O., Schechter, R.S. (eds.) Improved Oil Recovery by Surfactant and Polymer Flooding, pp. 55-92.Academic Press, New York City (1977)

Yortsos, Y.C., Hickernell, F.J.: Linear stability of immiscible displacement in porous media. SIAM J. Appl. Math. 49(3), 730-748 (1989) 